ARTICLE

Received 18 Jan 2016 | Accepted 1 Sep 2016 | Published 11 Oct 2016

DOI: $10.1038 /$ ncomms13099

OPEN

\title{
Salicylic acid receptors activate jasmonic acid signalling through a non-canonical pathway to promote effector-triggered immunity
}

Lijing Liư ${ }^{1}$, Fathi-Mohamed Sonbol ${ }^{1,2}$, Bethany Huot ${ }^{3}$, Yangnan $\mathrm{Gu}^{1}$, John Withers ${ }^{1}$, Musoki Mwimba ${ }^{1}$, Jian Yao ${ }^{4,5}$, Sheng Yang $\mathrm{He}^{4} \&$ Xinnian Dong ${ }^{1}$

It is an apparent conundrum how plants evolved effector-triggered immunity (ETI), involving programmed cell death (PCD), as a major defence mechanism against biotrophic pathogens, because ETI-associated PCD could leave them vulnerable to necrotrophic pathogens that thrive on dead host cells. Interestingly, during ETI, the normally antagonistic defence hormones, salicylic acid (SA) and jasmonic acid (JA) associated with defence against biotrophs and necrotrophs respectively, both accumulate to high levels. In this study, we made the surprising finding that JA is a positive regulator of RPS2-mediated ETI. Early induction of JA-responsive genes and de novo JA synthesis following SA accumulation is activated through the SA receptors NPR3 and NPR4, instead of the JA receptor COI1. We provide evidence that NPR3 and NPR4 may mediate this effect by promoting degradation of the JA transcriptional repressor JAZs. This unique interplay between SA and JA offers a possible explanation of how plants can mount defence against a biotrophic pathogen without becoming vulnerable to necrotrophic pathogens.

\footnotetext{
${ }^{1}$ Howard Hughes Medical Institute-Gordon and Betty Moore Foundation, Department of Biology, Duke University, Durham, North Carolina 27708, USA.

${ }^{2}$ Department of Basic Sciences, Faculty of Dentistry, Sinai University, Al Arish, North Sinai 45518, Egypt. ${ }^{3}$ Department of Energy Plant Research Laboratory, and Cell and Molecular Biology Program, Michigan State University, East Lansing, Michigan 48824, USA. ${ }^{4}$ Howard Hughes Medical Institute, Department of Energy Plant Research Laboratory, and Department of Plant Biology, Michigan State University, East Lansing, Michigan 48824, USA. ${ }^{5}$ Department of Biological Sciences, Western Michigan University, Kalamazoo, Michigan 49008, USA. Correspondence and requests for materials should be addressed to X.D. (email: xdong@duke.edu).
} 
$\mathrm{P}$ lants are constantly exposed to a wide range of microbial pathogens and herbivorous insects with different and sometimes opposing infection mechanisms. Therefore, they have to adjust their defence strategies accordingly through complex interplay between different phytohormones. Of those, salicylic acid (SA) is a major defence hormone against biotrophic and hemibiotrophic pathogens, which rely on living plant tissue for nutrients ${ }^{1-3}$. In contrast, plants produce jasmonic acid (JA) in response to wounding caused by insects and to necrotrophic microbes which obtain nutrients from dead host cells $^{3-5}$. The crosstalk between SA and JA was first observed in tomato where SA- or acetyl-SA treatment blocked not only JA production but also JA-mediated transcription of protease inhibitors involved in defence against insects ${ }^{6,7}$. Co-treating Arabidopsis with SA and JA abolished JA-mediated induction of the defensin gene PDF1.2 (refs 8,9), while infection by the hemibiotrophic bacterial pathogen Pseudomonas syringae. pv. tomato (Pst) DC3000, which enhanced SA production, led to reduced resistance to the necrotrophic fungal pathogen Alternaria brassicicola in the neighbouring tissue ${ }^{10}$. Conversely, mutants deficient in JA signalling were found to be more resistant to Pst DC3000 (refs 11,12). These studies clearly support the existence of SA-JA antagonism in basal resistance as a mechanism to prioritize the defence strategy according to the type of pathogen encountered. At the molecular level, this antagonism depends on the SA-signalling component nonexpressor of PATHOGENESIS-RELATED GENE 1 (NPR1) and the downstream transcription factors TGAs and WRKYs ${ }^{9,13-}$ 15; and the JA-inducible NAC transcription factors regulating SA biosynthesis and metabolism ${ }^{16}$.

Despite this well-documented reciprocal inhibition, the relationship between SA and JA is not always antagonistic. Studies in rice demonstrated that JA signalling positively regulates plant resistance to the biotrophic pathogen, Xanthomonas oryzae pv. oryzae $(X o o)^{17}$, possibly due to a common defence system activated by both SA and $\mathrm{JA}^{18}$. Mur et al. found that the outcome of the SA-JA interaction depends on relative concentrations of the two hormones ${ }^{19}$. Moreover, the antagonism between Pst DC3000 and A. brassicicola was not observed if Pst DC3000 carried the avrRpt2 effector gene ${ }^{10}$, which causes effector-triggered immunity (ETI) in the infected tissues. During this ETI induction, a high level of SA was produced, but no crosstalk suppression on JA signalling was detected ${ }^{10}$.

ETI is a major innate immune mechanism in plants against biotrophic pathogens that occurs when the activity of a pathogen effector delivered into the host cell is detected by a host nucleotide-binding leucine-rich repeat (NB-LRR) immune receptor $^{20,21}$. ETI triggers a dramatic immune response that often leads to sacrifice of the infected cells in the form of programmed cell death (PCD). In parallel to SA accumulation, the endogenous JA level also increases during ETI induction ${ }^{19,22}$, which may explain why ETI-associated PCD does not enhance plant susceptibility to necrotrophic pathogens in the neighbouring tissue $^{10}$. Understanding the cooperative interplay between SA and JA during ETI is, therefore, essential for elucidating the molecular mechanism of this key immune response in plants.

While the role of JA in ETI remains to be investigated, the molecular function of SA in ETI has been illustrated by the discovery of SA receptors, NPR3 and NPR4 (NPR3/4), which are substrate adaptors for Cullin 3 (Cul3) ubiquitin E3 ligases. The high concentration of SA generated during ETI has been shown to promote the degradation of NPR1 (ref. 23). Even though NPR1 is a positive regulator of SA-mediated basal resistance, it is a repressor of ETI ${ }^{23,24}$. During ETI, degradation of NPR1 is mediated by NPR3 whose interaction with NPR1 depends on high levels of $\mathrm{SA}^{23}$. A failure to remove
NPR1 repression in npr3 explains the ETI deficiency observed in this mutant. However, NPR1 may not be the only repressor that needs to be removed for ETI activation because the mutant of another SA receptor, NPR4, which does not interact with NPR1 at high levels of SA, was similarly deficient in ETI, and the npr3 npr4 double mutant had a more severe ETI deficiency than the single mutants ${ }^{23}$. Moreover, mutating NPR1 in npr3 npr4 could not fully restore ETI and PCD in the npr1 npr3 npr4 triple mutant, suggesting that NPR3 and NPR4 have additional substrates, besides NPR1, that are involved in this major immune response.

Similar to the SA receptors NPR3 and NPR4, which are adaptors for the Cul3 E3 ubiquitin ligase, the canonical JA receptor complex contains COI1 (CORONATINE INSENSITIVE 1), an F-box protein for the Cul1 E3 ubiquitin ligase, and one of the 12 JAZ (JASMONATE ZIM DOMAIN) proteins ${ }^{25}$. Interaction between COI1 and JAZs requires the presence of the biologically active (+)-7-iso-jasmonoyl-L-isoleucine $(\mathrm{JA}-\mathrm{Il})^{26}$. On perception of JA-Ile, JAZ proteins are ubiquitinated by SCFCOI1 and degraded by the $26 \mathrm{~S}$ proteasome $e^{11,27}$ to release their repression on transcription factors, such as MYCs, which are activators of JA-responsive genes $^{28,29}$. Although the coil-1 mutant has been reported to display enhanced resistance to Pst DC3000/avrRpm1, it was hypothesized to be due to the high basal defence in this mutant ${ }^{30}$. Thus, whether and how these JA signalling components are involved in the ETI-specific response needs to be explored.

In this study, we show that JA signalling positively regulates the establishment of RPS2-mediated ETI. However, the initial activation of JA-responsive genes during ETI is dependent on SA and SA receptors NPR3 and NPR4, but not on NPR1 or the canonical JA receptor COI1. ETI-mediated de novo JA synthesis is also compromised in an npr3 npr4 mutant. We further showed that NPR3 and NPR4 can interact with JAZ proteins in a manner that is enhanced by SA, and promote the reduction of JAZ1 protein levels at the early stage of ETI. This unique mechanism of reducing JAZ levels via NPR3/NPR4 provides an explanation for the observations above. Our study, therefore, reveals a new and intricate interplay between the two major plant defence hormone pathways.

\section{Results}

JA synthesis and signalling positively regulate ETI. To test our hypothesis that the JA signalling pathway is an additional level of regulation during ETI, we first examined the expression of several primary JA-responsive genes ${ }^{31}$ at $4 \mathrm{~h}$ post inoculation (h.p.i.) by Ps pv. maculicola (Psm) ES4326 carrying the effector avrRpt2 (ref. 32). As shown in Fig. 1a, five of the six genes tested were induced by Psm ES4326/avrRpt2 in wild-type (WT) Arabidopsis plants. In comparison, the induction of these genes was drastically compromised in the immune receptor mutant, rps2, which cannot recognize the activity of avrRpt2 (ref. 32), while the basal expression levels of these genes in WT and rps 2 were comparable (Supplementary Fig. 1). This dependence on immune receptor is not specific to RPS2, a CC-NB-LRR receptor ${ }^{33}$; a similar effect was also observed for the TIR-NB-LRR immune receptor RPS4 based on analysis of a previously published microarray data set $^{34}$ (Supplementary Fig. 2). JA-responsive genes were induced in 35S:RPS4-HS in the WT background after treatment, while in the eds1 mutant background compromised in RPS4-mediated ETI, the activation of these genes was blocked.

To further reveal the function of JA signalling during ETI, we sprayed plants with Methyl-JA (MeJA) $3.5 \mathrm{~h}$ after Psm ES4326/avrRpt2 infiltration and monitored ETI-associated PCD by measuring ion leakage (an indicator of cell death) during a 24-h time course. We observed that MeJA could further accelerate 


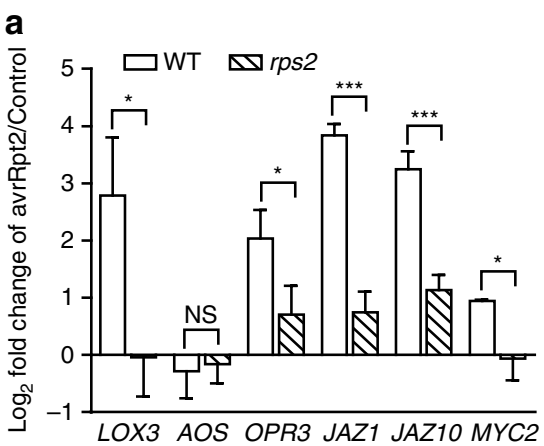

c
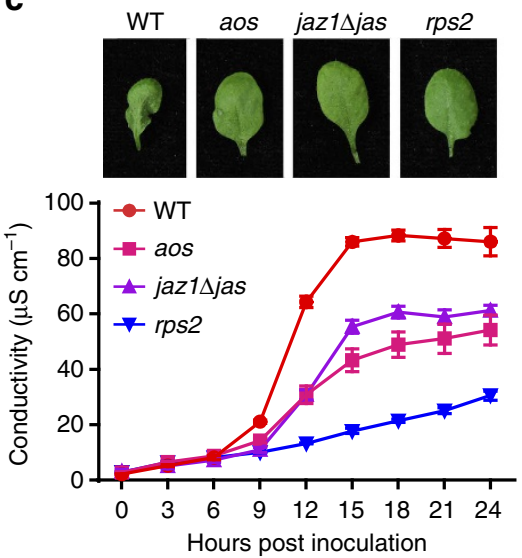

e

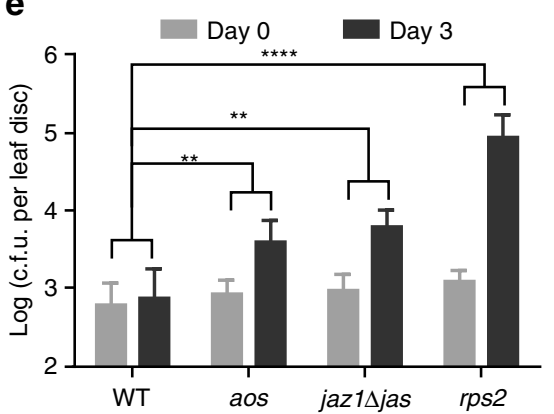

b

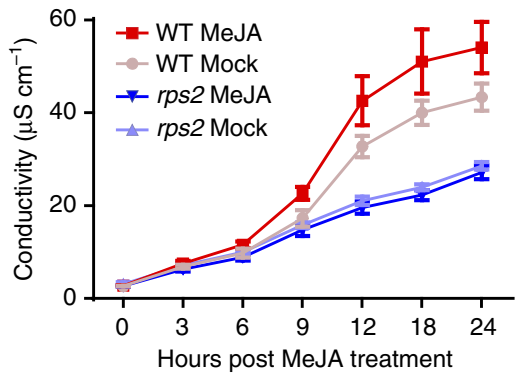

d
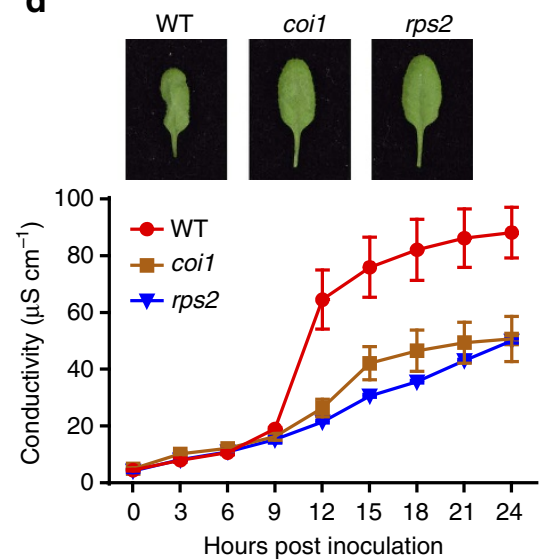

f

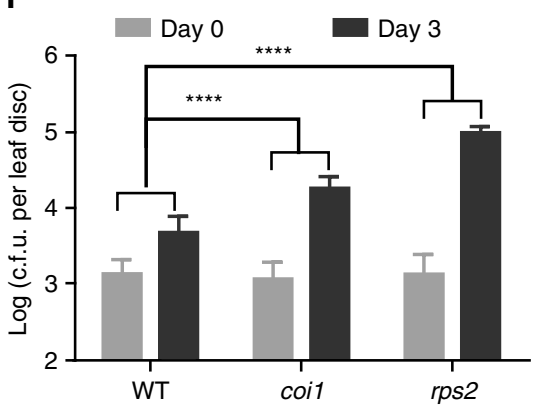

Figure 1 | JA synthesis and signalling are activated through the RPS2 immune receptor and required for ETI and associated PCD. (a) Expression levels of JA-responsive genes in wild-type (WT) and the rps2 mutant at $4 \mathrm{~h}$ post inoculation (h.p.i.) with Psm ES4326/avrRpt2 at OD $600 \mathrm{~nm}=0.2$. The final data were normalized to the expression with $10 \mathrm{mM} \mathrm{MgSO}_{4}$ (Control) treatment. qRT-PCR was performed on LOX3 (LIPOXYGENASE 3), AOS (ALLENE OXIDE SYNTHASE), OPR3 (OPDA REDUCTASE 3), JAZ1 ( JASMONATE ZIM-DOMAIN PROTEIN 1), JAZ10, MYC2 (ARABIDOPSIS MYELOCYTOMATOSIS ONCOGENE HOMOLOG 2) with UBQ5 (UBIQUITIN 5) as a reference. Data from three biological replicates were combined using linear mixed-effects model. Significant difference was detected using Student's $t$-test. Data are presented as Mean \pm s.d. (b) Three-week-old plants were first infiltrated with Psm ES4326/avrRpt2 at $\mathrm{OD}_{600 \mathrm{~nm}}=0.01$, and $3.5 \mathrm{~h}$ later water (Mock) or $100 \mu \mathrm{M} \mathrm{MeJA}$ (MeJA) was sprayed. Starting at $0.5 \mathrm{~h}$ post inoculation (h.p.i.), leaf discs were collected for conductivity assay. Data are shown as mean \pm s.d. ( $n=3$ biological replicates). (c) Representative leaves of WT, aos, jaz14jas, and rps2 plants 15 h.p.i. by Psm ES4326/avrRpt2 at $\mathrm{OD}_{600 \mathrm{~nm}}=0.01$ (upper panel). Conductivity measurements were performed 0.5 h.p.i. with Psm ES4326/avrRpt2 (lower panel). Data are shown as mean \pm s.d. ( $n=3$ biological replicates). (d) Representative leaves and conductivity measurements of WT, coi1 and rps 2 plants. The pathogen inoculation and conductivity measurements were as described in c. Data are shown as mean \pm s.d. ( $n=3$ biological replicates). (e) Plants were infiltrated with Psm ES4326/avrRpt2 at $\mathrm{OD}_{600 \mathrm{~nm}}=0.002$ and pathogen growth was measured at day 0 and day 3. c.f.u., colony forming unit. Significant difference was detected by two-way ANOVA. Data are shown as mean \pm s.d. ( $n=8$ biological replicates). (f) The growth of Psm ES4326/avrRpt2 in WT, coil, and rps2. The same method was used as described in e. All experiments were repeated three times with similar results. ${ }^{\star} P<0.05 ;{ }^{\star \star} P<0.01$;

${ }^{\star \star \star} P<0.001 ;{ }^{\star \star \star} P<0.0001$; NS, no significant difference. $\mathrm{QRT}$-PCR, quantitative reverse transcription PCR.

PCD in WT plants, but not in the rps2 mutant (Fig. 1b). Next, we measured ion leakage in three JA mutants: aos (ALLENE OXIDE SYNTHASE), a JA-deficient mutant ${ }^{35,36}$; coil, the JA receptor mutant ${ }^{25}$; and jaz1 1 jas, a transgenic line over-expressing a JAZ1 protein that lacks the Jas domain and exhibits a constitutive repressor activity on JA signalling ${ }^{11}$. Homozygous coil plants were selected on MS media supplemented with JA (Supplementary Fig. 3). These mutants all showed compromised PCD compared to
WT on Psm ES4326/avrRpt2 challenge (Fig. 1c, d), consistent with a positive role of JA in ETI. In addition, resistance triggered by the avrRpt2 effector was also diminished in aos, coil and jaz14jas in comparison to the WT control (Fig. 1e,f). Collectively, these data demonstrate that JA synthesis and signalling are positive regulators of RPS2-mediated ETI.

According to our results in Fig. 1, we made the assumption that during ETI, de novo JA synthesis occurs before effector-triggered 
PCD which is normally observed after 12 h.p.i. To test this hypothesis, we measured the concentrations of free SA, SA $\mathrm{O}-\beta$-glucoside (SAG), JA-Ile and JA at $0,4,8$, and $12 \mathrm{~h}$ after infiltrating WT and rps2 with Psm ES4326/avrRpt2. As shown in Fig. 2, in response to pathogen inoculation, there were a sequential increase in these measured hormones with free SA starting as early as 4 h.p.i. (Fig. 2a), total SA (SA+SAG) and JA at 8 h.p.i. (Fig. 2b,d), and then JA-Ile at 12 h.p.i. (Fig. 2c). These increases were significantly dampened in the rps2 mutant, indicating that they were ETI-specific. We also observed an ETI-induced ABA accumulation initiating at 4 h.p.i. (Supplementary Fig. 4).

Early JA induction in ETI is principally dependent on NPR3/4. Since SA and JA are sequentially induced during ETI, we next examined a possible connection between these two hormones by analysing the expression of JA-responsive genes 4 h.p.i. by Psm ES4326/avrRpt2 in the SA-deficient sid2 mutant ${ }^{2}$. Contrary to the expected antagonism of SA on the JA response, the induction of $J A Z 1, J A Z 10$ and LOX3 was inhibited in the sid2 mutant similar to the level observed in the rps2 mutant (Fig. 3a). These data showed that SA is a necessary signal for this early induction of JA signalling during RPS2-mediated ETI. As NPR1 has been proposed as an SA receptor ${ }^{37,38}$, and a major regulator of both SA signalling ${ }^{1}$ and SA-mediated repression of the JA response ${ }^{9}$, we next tested whether NPR1 is also involved in this SA-dependent JA signalling activation at the early stage of ETI. Our result showed that mutating NPR1 had little effect on the ETI-triggered induction of these JA-responsive genes (Fig. 3b).

On the basis of the report that NPR3 and NPR4 are the SA receptors functionally required for $\mathrm{ETI}^{23}$, we analysed the expression of JA-responsive genes in the npr3 npr4 double mutant. We found that in the npr3 npr4 mutant, the early induction of $J A Z 1, J A Z 10$ and $L O X 3$ at 4 h.p.i. was compromised (Fig. 3c). This result indicates that the activation of JA signalling at the early stage of RPS2-mediated ETI principally depends on
NPR3/4, but is independent of NPR1. Consistent with this conclusion, comparable expression of JA-responsive genes was detected in npr3 npr4 and npr1 npr3 npr4 (Fig. 3c). The basal expression of these JA-responsive genes and the SA-mediated repression of JA-induced PDF1.2 expression were intact in the npr3 npr4 mutant (Supplementary Figs 5 and 6), indicating that NPR3/4 affect JA signalling only during ETI.

Activation of the canonical JA signalling pathway involves degradation of JAZs through the JA receptor COI1, an F-box protein of an SCF-type E3 ubiquitin ligase ${ }^{11,39}$. To test if ETImediated early induction of JA signalling is dependent on de novo JA synthesis and canonical JA signalling, we checked the expression of JA-responsive genes in the aos, coil and jaz14jas mutants. Surprisingly, the induction of JA-responsive genes at 4 h.p.i. by Psm ES4326/avrRpt2 was abolished only in jaz1 1 jas, but not in aos or coil (Fig. 3d; Supplementary Fig. 7). On the contrary, the induction of these genes was even higher in aos than in WT, likely due to the higher level of SA in JA mutant ${ }^{12}$. These results indicate that the ETI-mediated early induction of JA signalling is independent of de novo JA synthesis, but dependent on the degradation of JAZs, and occurs through a non-canonical pathway in which NPR3 and NPR4, instead of COI1, play an essential role.

Interactions between NPR3/4 and JAZs are enhanced by SA. JAZ proteins are the central transcriptional repressors of JA signalling ${ }^{27,40}$. On the basis of our discovery that NPR3/4 are required for early activation of JA signalling during RPS2-mediated ETI, we hypothesized that these proteins might serve as adaptors for an E3 ubiquitin ligase that targets the JAZ proteins for degradation. To test our hypothesis, we first performed yeast two-hybrid (Y2H) assays. Each of the twelve JAZ proteins was fused with the transcriptional activation domain (AD), and NPR3 and NPR4 were fused with the DNA binding domain (BD). As shown in Fig. 4a, nine of the $12 \mathrm{JAZ}$ proteins interacted with NPR3. Six of these nine interactions required the presence of SA (JAZ1, 2, 5, 8, 10, 11), two were enhanced by SA
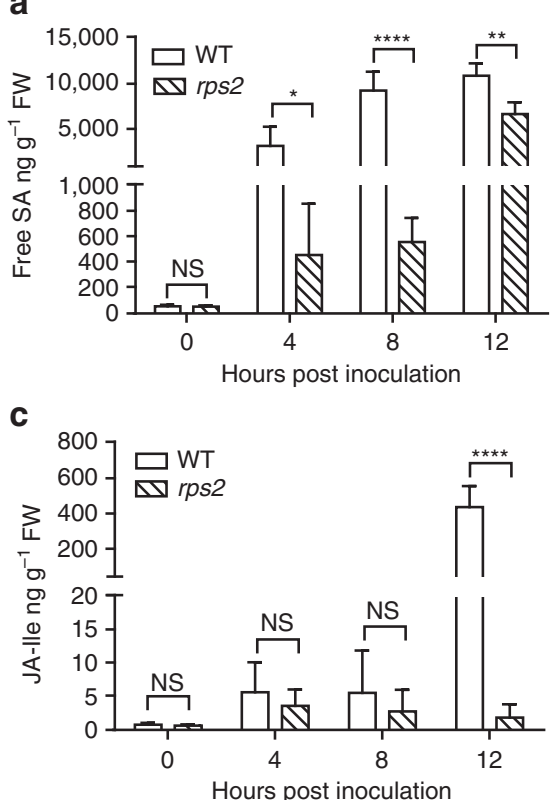

b

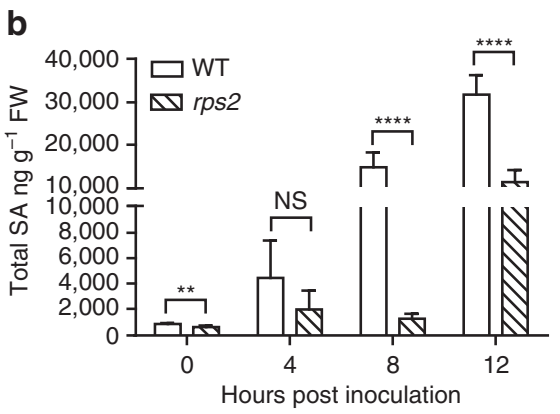

d

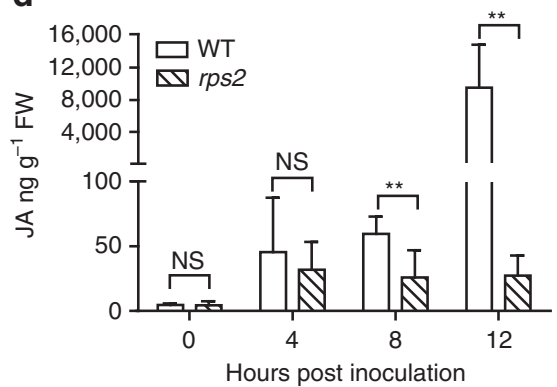

Figure 2 | The levels of SA and JA in WT and rps2 during ETI. Three-week-old WT and rps2 plants were infiltrated with Psm ES4326/avrRpt2 at $\mathrm{OD}_{600 \mathrm{~nm}}=0.01$. Samples were collected at 0, 4, 8, 12 h.p.i. The levels of (a) free SA; (b) total SA; (c) JA-lle; and (d) JA were measured. Significant difference was detected using Student's $t$-test. Data are shown as mean \pm s.d. ( $n=5-6$ biological replicates). All experiments were repeated twice with similar results. ${ }^{\star} P<0.05 ;{ }^{\star \star} P<0.01 ;{ }^{\star \star \star \star} P<0.0001 ;$ NS, no significant difference. 
a

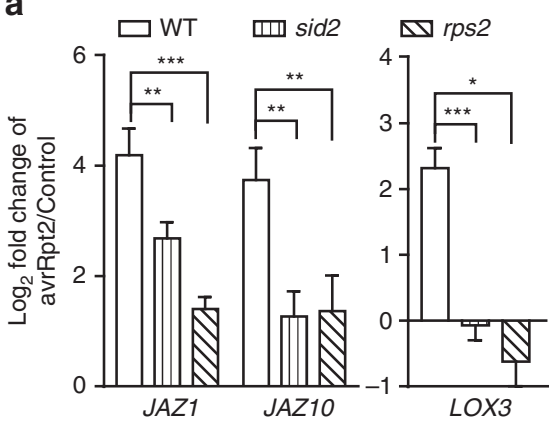

C

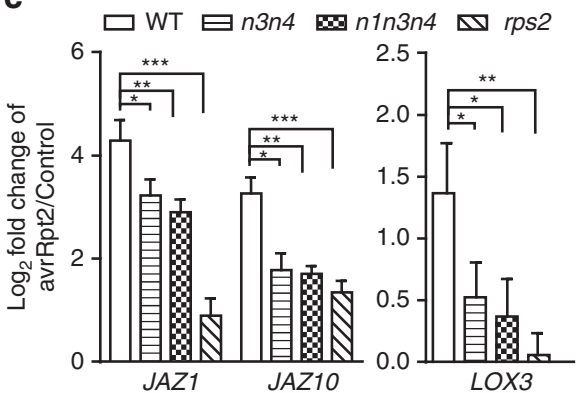

b

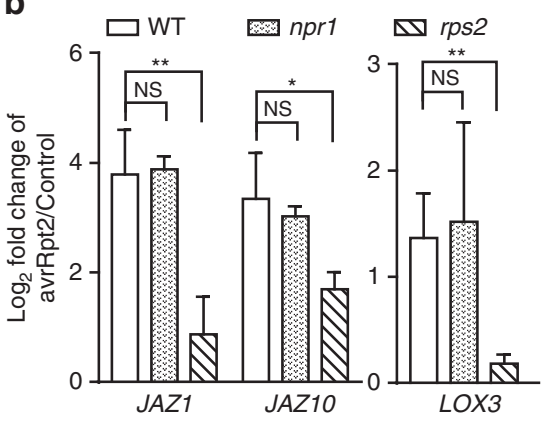

d

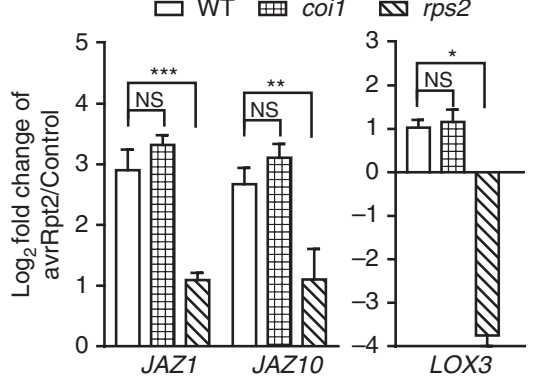

Figure 3 | ETI-mediated early induction of JA-responsive genes is dependent on SA and NPR3 and NPR4 but independent of NPR1 and the JA receptor COI1. Leaves from corresponding plants were harvested 4 h.p.i. with Psm ES4236/avrRpt2 (avrRpt2) at $\mathrm{OD}_{600 \mathrm{~nm}}=0.2$ or $10 \mathrm{mM} \mathrm{MgSO} 4$ (Control). qRT-PCR was performed on LOX3, JAZ1, JAZ10, with UBQ5 as a reference. Gene expression (a) in WT, sid2 and rps2; (b) in WT, npr1 and rps2; (c) in WT, npr3 npr4 (n3n4), npr1 npr3 npr4 (n1n3n4) and rps2; (d) in WT, coi1 and rps2 was measured. Data from three biological replicates were combined using linear mixed-effects model. Significant difference was detected using Student's $t$-test. Data are shown as mean \pm s.d. ${ }^{\star} P<0.05 ;{ }^{\star \star} P<0.01 ;{ }^{\star \star \star} P<0.001 ;$ NS, no significant difference. qRT-PCR, quantitative reverse transcription PCR.

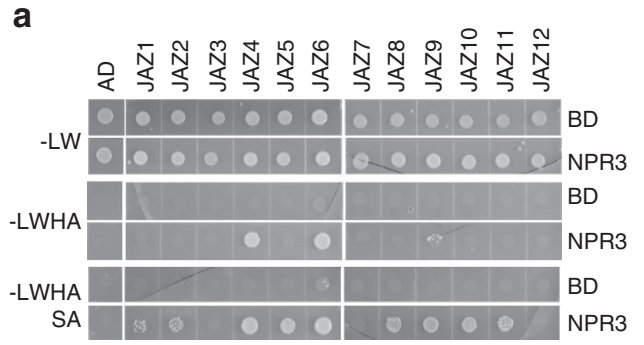

b

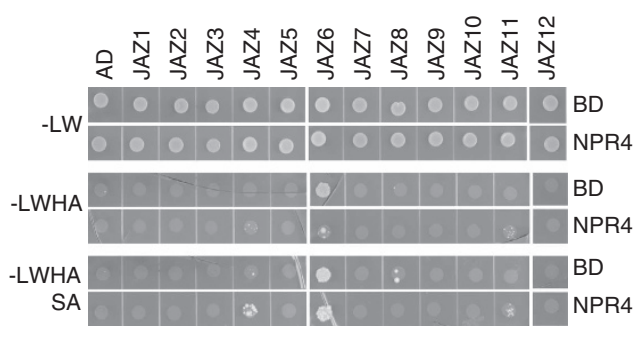

C

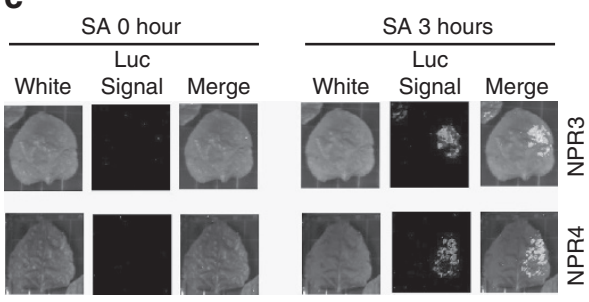

d

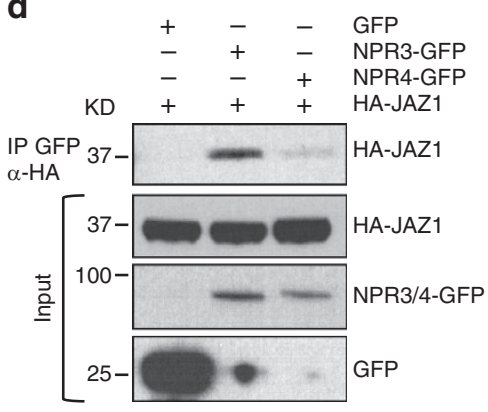

Figure 4 | SA facilitates NPR3 and NPR4 interactions with JAZ proteins. Yeast two-hybrid assay (Y2H) was performed to study (a) interactions between NPR3 and JAZs and (b) interactions between NPR4 and JAZs. For SA treatment, $100 \mu \mathrm{M} \mathrm{SA}$ was added in the yeast media. (c) The split-luciferase assays were performed on JAZ1 with NPR3 or NPR4. The left half of the N. benthamiana leaf was co-infiltrated with cLuc and NPR3-nLuc or cLuc and NPR4-nLuc as control. The right half of the leaf was co-infiltrated with cLuc-JAZ1 and NPR3-nLuc or cLuc-JAZ1 and NPR4-nLuc. After two days of incubation, luciferin was sprayed onto the inoculated leaves and chemiluminescence images were taken by a CCD camera 0 and $3 \mathrm{~h}$ after $1 \mathrm{mM}$ SA treatment.

(d) The co-immunoprecipitation (co-IP) assay on JAZ1 with NPR3 or NPR4 in Arabidopsis. Samples were collected at 4 h.p.i. with Psm ES4326/avrRpt2, at $\mathrm{OD}_{600 \mathrm{~nm}}=0.2$, plus $40 \mu \mathrm{M}$ MG115. The co-IP assay was carried out using the GFP-Trap_A beads for $2 \mathrm{~h}$ at $4{ }^{\circ} \mathrm{C}$. The HA-JAZ1 protein was detected by western blot using HA antibody. The NPR3-GFP, NPR4-GFP or GFP protein levels were measured by western blots using the GFP antibody. All experiments were repeated three times with similar results. 
(JAZ4 and JAZ9) and one was SA independent (JAZ6). In addition to NPR3, two JAZ proteins (JAZ4 and JAZ11) were also found to interact with NPR4 (Fig. 4b).

We next used a split luciferase assay to verify these interactions in planta. JAZ1 and NPR3 were fused with C-terminal (cLuc) and $\mathrm{N}$-terminal (nLuc) halves of luciferase, respectively, to generate cLuc-JAZ1 and NPR3-nLuc, and were transiently co-expressed in leaves of Nicotiana benthamiana. The complemented luciferase activity was detected with the combination of cLuc-JAZ1 and NPR3-nLuc after SA treatment (Fig. 4c upper panel; Supplementary Fig. 8a). Even though NPR4 could not interact with JAZ1 in yeast, it showed a similar interaction pattern with JAZ1 in the split luciferase assay (Fig. 4c lower panel; Supplementary Fig. 8b). The same assay was applied to JAZ4, 6, 8 and 9 and all of them showed SA-enhanced interactions with both NPR3 and NPR4 (Supplementary Fig. 8). The SA-dependency for most of the NPR4-JAZ interactions is surprising because unlike NPR3, NPR4 interaction with NPR1 is constitutive until disrupted by $\mathrm{SA}^{23}$. However, this result is consistent with our hypothesis that both NPR3 and NPR4 can serve as ubiquitin E3 ligase adaptors for substrates other than NPR1.

To further confirm NPR3/4-JAZ1 interactions, we performed coimmunoprecipitation (co-IP) and pull down assays. We found that when JAZ1 was co-expressed with GFP, NPR3-GFP or NPR4-GFP in N. benthamiana leaves or in Arabidopsis transgenic lines, it was able to co-IP with NPR3 and NPR4 (Fig. 4d; Supplementary Fig. 9). To study the effect of SA on these interactions, we expressed NPR3-GFP and NPR4-GFP proteins in the $N$. benthamiana transgenic line carrying the salicylate hydroxylase gene, NahG. As shown in Supplementary Fig. 10, SA, but not JA, could enhance the pulldown of NPR3-GFP and NPR4-GFP by the purified recombinant GST-JAZ1. Interestingly, the NPR3-GFP and NPR4-GFP pulled down by GST-JAZ1 had higher-molecular weights than expected (Supplementary Fig. 10), probably due to unknown modifications. Collectively these data demonstrated that NPR3 and NPR4 could bind to JAZ proteins and SA could enhance the binding.

To determine which domains of JAZ1 and NPR3 are responsible for their interaction, we made truncated versions of both proteins. For JAZ1, we generated individual deletions of each of the three protein interacting domains, namely the $\mathrm{N}$-terminal domain (NT), the zinc-finger expressed in inflorescence meristem domain (ZIM) and the jasmonate-associated domain (Jas) ${ }^{41}$. For NPR3, we separately removed the Bric-aBrac, Tramtrack, Broad-complex (BTB) domain and the ankyrin repeat (ANK) domain, known to be involved in protein-protein interaction ${ }^{23}$, and the $\mathrm{N}$ - and C-terminal regions (Supplementary Fig. 11a). These JAZ1 and NPR3 constructs were then fused with the $\mathrm{AD}$ and $\mathrm{BD}$ domain, respectively, for $\mathrm{Y} 2 \mathrm{H}$ experiments. We found that even though all of the mutant proteins expressed well in yeast (Supplementary Fig. 11b), deleting any of the domains in JAZ1 or NPR3 compromised their interaction, except deletion of the N-terminal region of NPR3, which made the interaction constitutive (Supplementary Fig. 11c). These data showed that the overall protein integrity for both JAZ1 and NPR3 is important for their interaction while the N-terminal region of NPR3 may impose an inhibitory effect.

NPR3/4 promote the reduction of JAZ1 levels during ETI. The deficiency in RPS2-mediated ETI observed in the jaz14jas transgenic line (Fig. 1c,e) might be due to stabilization of the mutant JAZ1 repressor in the absence of the Jas domain, which is required for interaction with its canonical partner, COI1 (ref. 39). Alternatively, the phenotype might result from a lack of interaction with NPR3/4, which might also affect JAZ1 stability, albeit through a different E3 ubiquitin ligase complex (that is, Cullin 3 instead of Cullin 1). To test which hypothesis is true, we first demonstrated that JAZ1 is a repressor of ETI by over-expressing (OE) HA-tagged JAZ1. We found that in response to Psm ES4326/avrRpt2 inoculation, the 35S:HA-JAZ1 OE plants were impaired in effector-triggered PCD (Fig. 5a) similar to the jaz1 4 jas transgenic line (Fig. 1c).

On the basis of our findings that both NPR3 and NPR4 interact with JAZ proteins in an SA-enhanced manner and the npr 3 npr4 double mutant has a more severe ETI deficiency than the npr3 and npr4 single mutants, we proposed that NPR3 and NPR4 function redundantly to degrade JAZ proteins during $\mathrm{ETI}^{23}$. To test this, we crossed the 35S:HA-JAZ1 OE line into the npr3 npr4 and the rps2 mutant backgrounds, respectively, and examined the effects that these mutations had on the constitutively expressed HA-JAZ1. Without pathogen challenge, neither the HA-JAZ1 mRNA nor the HA-JAZ1 protein was affected by the npr 3 npr4 or $r p s 2$ mutations (Supplementary Fig. 12). However, $4 \mathrm{~h}$ after Psm ES4326/avrRpt2 challenge, HA-JAZ1 protein levels were approximately $50 \%$ lower in the WT background, but not in the immune receptor mutant rps2 (Fig. 5b; Supplementary Fig. 13). Importantly, this reduction in JAZ1 level was not seen in the npr3 npr4 mutant with the WT level of COI1 protein (Fig. 5b; Supplementary Figs 13 and 14). The trigger for this NPR3/4-mediated JAZ1 reduction is SA-dependent, because in the SA-deficient sid2 mutant, HA-JAZ1 remained stable at 4 h.p.i. by Psm ES4326/avrRpt2 (Fig. 5c). Interestingly, exogenous application of SA was not sufficient to promote the reduction of JAZ1 level through NPR3 and NPR4 indicating that the SA accumulation during ETI is specifically required (Fig. 5d; Supplementary Fig. 15a).

To test whether this NPR3/4-dependent reduction of JAZ1 protein levels during ETI is dependent on the 26S proteasome, we infiltrated MG115 together with Psm ES4326/avrRpt2 and found that addition of MG115 completely blocked the reduction in JAZ1 levels during ETI (Fig. 5b). Quantification of protein levels showed that the JAZ1 protein in the MG115-treated sample accumulated to $>100 \%$ of the $\mathrm{MgSO}_{4}$-treated control (Supplementary Fig. 13), consistent with the observation that a JAZ1 levels were also reduced non-specifically as a result of the inoculation procedure (Supplementary Fig. 16). We further confirmed that mutating both NPR3 and NPR4 did not affect JAZ1 degradation triggered by exogenous application of JA (Supplementary Fig. 15b). These results suggest that NPR3 and NPR4 can specifically promote the degradation of JAZ1 through the activity of the $26 \mathrm{~S}$ proteasome at the early stage of RPS2mediated ETI.

$\mathrm{NPR} 3 / 4$ are required for ETI-triggered de novo JA synthesis. To test whether the NPR3/4 are required for de novo JA synthesis during ETI (Fig. 2), we measured free SA, SAG, JA and JA-Ile levels in the npr3 npr4 mutant in response to Psm ES4326/avrRpt2. We found that in npr3 npr4, the total SA level was lower than in WT at 0 h.p.i., whereas the free SA level was less induced compared with WT at 12 h.p.i. (Supplementary Fig. 17). These results could be due to the accumulation of NPR1, a known repressor of SA synthesis ${ }^{42}$, in npr3 npr4 (ref. 23). Consistent with our hypothesis that de novo JA synthesis may be a consequence of early reduction of JAZ levels and induction of JA-responsive genes, we detected significantly lower JA and JA-Ile levels in the npr3 npr4 mutant in comparison with WT at 12 h.p.i. (Fig. 5e; Supplementary Fig. 17d). These data indicated that NPR3/4 promote de novo JA synthesis during RPS2-mediated ETI. 
a

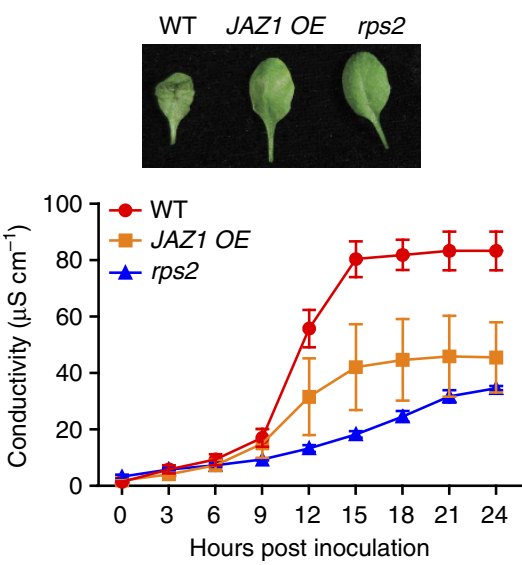

C

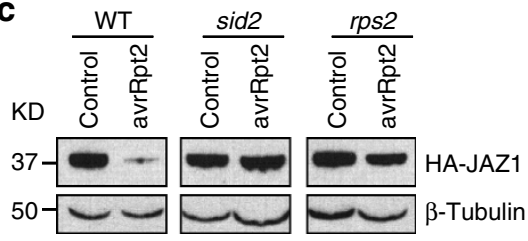

e

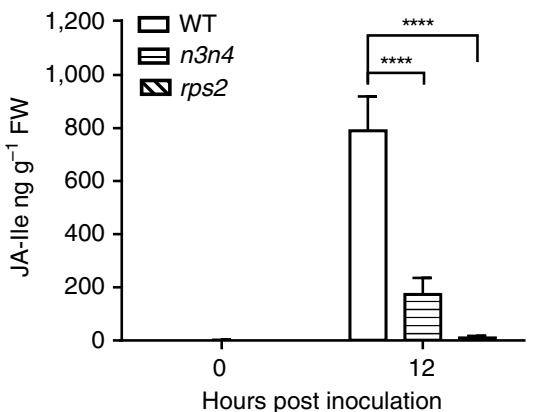

b

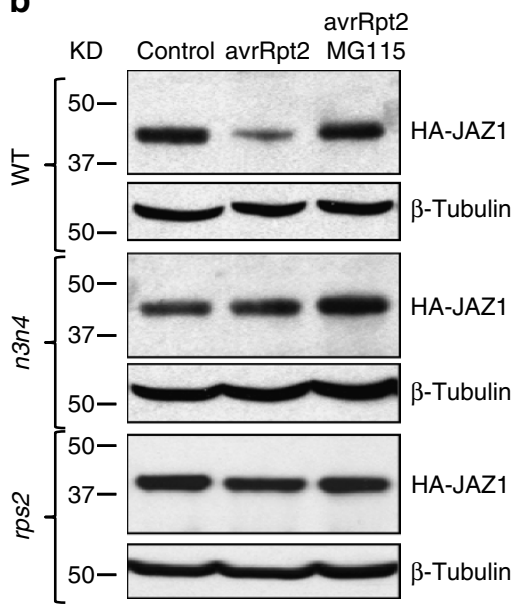

d

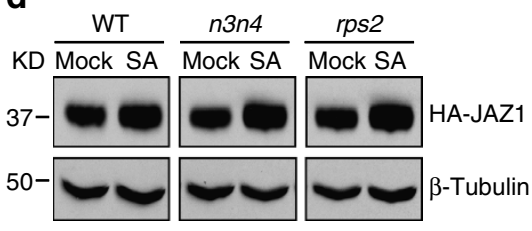

f

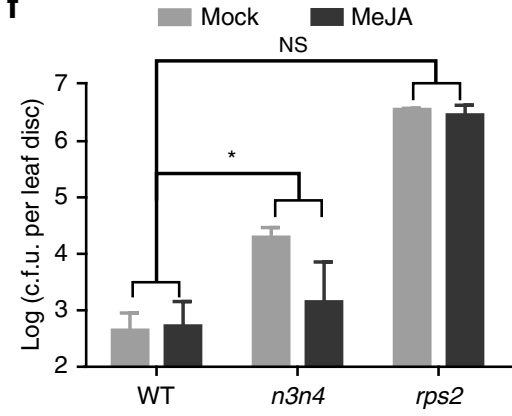

Figure 5 | NPR3 and NPR4 promote ETI-induced reduction of JAZ1 level and de novo JA synthesis. (a) Representative leaves and conductivity measurements of WT, the 35S:HA-JAZ1 overexpressing (JAZ1 OE) transgenic line, and the rps2 mutant. The same methods were used as in Fig. 1c. Data are shown as mean \pm s.d. ( $n=3$ biological replicates). (b) HA-JAZ1 protein levels were determined in WT, npr3 npr4 (n3n4) and the rps2 mutant 4 h.p.i. with $10 \mathrm{mM} \mathrm{MgSO}_{4}$ (Control), Psm ES4326/avrRpt2 (avrRpt2) or Psm ES4326/avrRpt2 together with $40 \mu \mathrm{M}$ MG115 (avrRpt2 MG115). (c) HA-JAZ1 protein levels were measured in WT, sid2 and the rps2 mutant 4 h.p.i. with $10 \mathrm{mM} \mathrm{MgSO}_{4}$ (Control), or Psm ES4326/avrRpt2 (avrRpt2). (d) HA-JAZ1 protein levels were determined in WT, npr3 npr4 (n3n4) and the rps2 mutant $4 \mathrm{~h}$ after being sprayed with water (Mock) or $1 \mathrm{mM}$ SA. For (b,c,d) the western blots were performed using the HA antibody. $\beta$-Tubulin served as a loading control. KD, kilodalton. (e) The JA-lle levels in WT, npr3 npr4 and rps2 at 0 and 12 h.p.i. with Psm ES4326/avrRpt2 at $\mathrm{OD}_{600 \mathrm{~nm}}=0.01$. Significant difference was detected using Student's $t$-test. Data are shown as mean $\pm \mathrm{s}$.d. ( $n=5-6$ biological replicates). (f) Plants were infiltrated with Psm ES4326/avrRpt2 at $\mathrm{OD}_{600 \mathrm{~nm}}=0.01$ and $3.5 \mathrm{~h}$ later water (Mock) or $100 \mu \mathrm{M} \mathrm{MeJA}$ (MeJA) was sprayed. Pathogen growth was measured at 1 day post inoculation. Significant difference was detected by two-way ANOVA. Data are shown as mean \pm s.d. ( $n=8$ biological replicates). All experiments were repeated three times with similar results. ${ }^{\star} P<0.05 ;{ }^{\star \star \star \star} P<0.0001$; NS, no significant difference.

To determine whether the lack of de novo JA synthesis and/or signalling is responsible for the compromised ETI in the npr3 $n$ pr4 mutant, we sprayed npr3 npr4 plants with MeJA at 3.5 h.p.i. with Psm ES4326/avrRpt2. We used a higher dose of Psm ES4326/ avrRpt2 for this experiment, because of the elevated basal resistance in the npr3 npr4 mutant due to the accumulation of NPR1 (ref. 23). MeJA application recovered resistance in the npr3 npr4 mutant, but did not further increase resistance in WT (Fig. 5f; Supplementary Fig. 18). The rescue of ETI in npr3 npr4 by the exogenous application of MeJA is likely through the activity of COI1 protein which is intact in the mutant (Supplementary Fig. 14). This result indicates that NPR3/ 4-mediated activation of early JA signalling and/or synthesis is essential for RPS2-mediated ETI and the canonical JA pathway is also important for the subsequent signal amplification.

\section{Discussion}

Through this study, we discovered a noncanonical mechanism of activating the JA signalling pathway through the SA receptors NPR3 and NPR4, instead of the canonical JA receptor COI1, in the early stage of ETI induction. This early JA signalling induction event is required for and amplified by the subsequent de novo JA synthesis and signalling. However, how the JA signalling pathway contributes to ETI in plants requires further investigation. 
SA is a necessary signal for the activation of JA signalling at the early stage of ETI (Fig. 3a), but whether SA is a sufficient signal will require further study because exogenous application of SA is known to repress the JA pathway instead of activating it. SA treatment alone could not lead to the degradation of JAZ1 protein as occurs following inoculation with Psm ES4326/avrRpt2 (Fig. 5d; Supplementary Fig. 15a). ETI may cause in planta accumulation of SA to a higher level than that achieved through exogenous application or in a specific subcellular compartment or trigger SA synthesis with a unique kinetic. Alternatively, during ETI, a co-activator may be produced for SA-mediated stimulation of JA signalling.

As summarized in Fig. 6, on ETI induction, multiple signalling pathways are activated. A major event of the induction is the accumulation of SA. The high level of SA at the infection site promotes NPR3-mediated degradation of NPR1 (ref. 23) to remove its repression on ETI (PCD) and alleviate its crosstalk inhibition on JA signalling, meanwhile we propose that degradation of JAZs by NPR3 and NPR4 directly activates JA signalling, which is further amplified by de novo JA synthesis. It is intriguing that SA inhibits the NPR4-NPR1 interaction ${ }^{23}$, but stimulates the NPR4-JAZs interaction (Fig. 4c; Supplementary Fig. 8), consistent with the ETI deficient phenotype observed in the npr4 mutant ${ }^{23}$.

The recovery of ETI resistance in the npr 3 npr4 mutant by the exogenous application of JA (Fig. 5f) indicates that the subsequent JA signal amplification through the canonical pathway is also important for this immune response. This explains the ETI-deficiency observed in the JA synthesis and signalling mutants (Fig. 1).

The synergy between SA and JA during ETI is distinct from the previously reported antagonism ${ }^{8,16,43}$, which was normally observed in the context of basal resistance ${ }^{10,11,15}$. Although it is

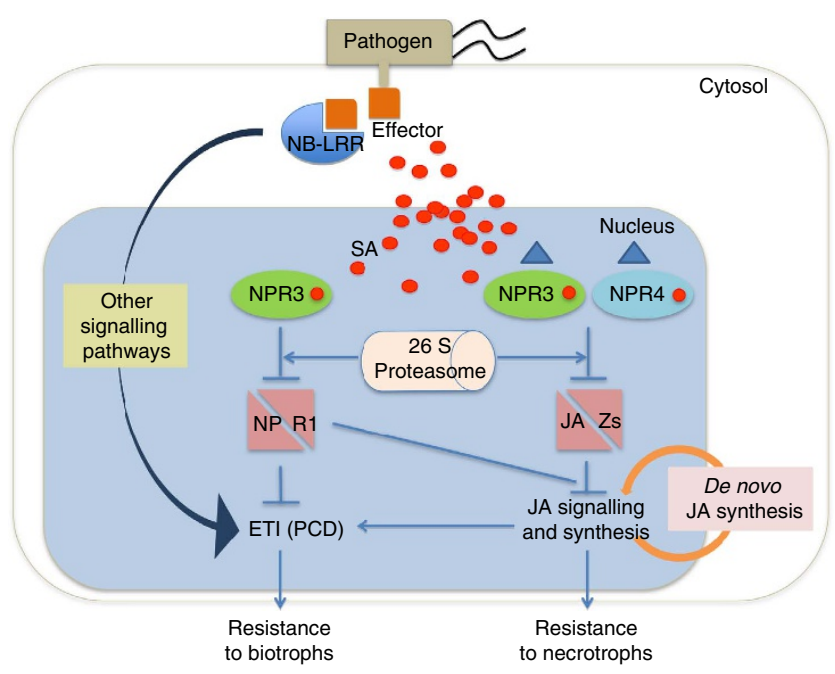

Figure 6 | Working model of the interplay between SA and JA during ETI. Activation of an NB-LRR immune receptor in plants by a pathogen effector leads to induction of multiple signalling pathways. A major event of the induction is the accumulation of SA at the infection site. At the high level of SA, NPR3 can interact with its substrate NPR1 to remove its repression on $\mathrm{ETI}$ and on crosstalk inhibition of the JA signalling pathway. Both NPR3 and NPR4 can also interact with JAZ proteins in an SA-enhanced manner leading to the degradation of JAZs. This results in activation of de novo JA synthesis of JA and amplification of the JA signalling through the canonical pathway. Activation of both SA- and JA-signalling pathways during ETI enables plants to use this PCD-associated defence strategy against biotrophic pathogens without making them vulnerable to necrotrophic pathogens. The blue triangle shape in the graph represents an unknown signal that may affect the degradation of JAZ1 through NPR3 and NPR4. not known how exactly these opposing relationships between the two defence hormones are regulated, the unique interplay between SA and JA during ETI supports the minority opinion ${ }^{44}$ that ETI is not an amplified and prolonged version of basal resistance but rather a distinct immune strategy that is only deployed when basal defence becomes insufficient.

Our study demonstrated a unique functional role for JA in ETI. The cooperation between SA and JA, two defence hormones against biotrophic and necrotrophic pathogens, provides a mechanism by which plants can use PCD as a major defence mechanism against biotrophic pathogens without making them vulnerable to necrotrophic pathogens ${ }^{10}$.

\section{Methods}

Plant materials. All the mutants used in this research were in the Columbia (Col-0) ecotype background. The mutants aos, sid2, npr1, npr3 npr4, npr1 npr3 npr4, rps2, coi1-30, the 35S:HA-JAZ1 line and the 35S:jaz1 1 jas line were described previously ${ }^{10,23,45,46}$. 35S:GFP, 35S:NPR3-GFP, 35S:NPR4-GFP were constructed using the pK7FWG2 vector and transformed to wild type. The 35S:HA-JAZ1 line was crossed with npr3 npr4, sid2 and rps2 to generate the 35S:HA-JAZ1/npr3 npr4, 35S:HA-JAZ1/sid2 and 35S:HA-JAZ1/rps2 lines, and crossed with 35S:GFP, 35S: NPR3-GFP, 35S:NPR4-GFP to generate 35S:HA-JAZ1/35S:GFP, 35S:HA-JAZ1/35S: NPR3-GFP, 35S:HA-JAZ1/35S:NPR4-GFP, respectively.

Quantitative real time PCR. Leaf tissues were collected from 3-week-old plants after the corresponding treatment at the indicated time points and total RNA was extracted using TRIzol Reagent according to the manufacture's instruction. Two microgram of the total RNA sample was treated with TURBO DNA-free (Cat No. AM1907, Invitrogen) to eliminate the genomic DNA contamination. Then cDNA was synthesized by SuperScript III reverse transcriptase (Cat No. 18080-044, Invitrogen) along with oligo-dT primers and analysed by quantitative reverse transcription PCR using SYBR Green Master with the gene specific primers listed in Supplementary Table 1. The level of UBQ5 was used to normalize the expression of target genes. Data analysis was performed using mixed linear models in the $\mathrm{R}$ programming environment ${ }^{47}$. Genotypes and treatments were used as fixed effects and replicate-specific effects were considered random effects. Modelled expression values between genotypes were compared using Student's $t$-test where indicated.

Split luciferase assay. Split luciferase assay was performed according to a classic method $^{48}$. JAZs were cloned into a pDEST vector with the C-terminal half of luciferase (cLuc) and NPR3 and NPR4 were cloned into another pDEST vector with the N-terminal half of luciferase (nLuc). They were then individually transformed into Agrobacterium tumfaciens GV3101 and transiently co-expressed in one half of each Nicotianna benthamiana leaf by infiltration ${ }^{49}$. As a control, the other half of each leaf was co-infiltrated with GV3101 carrying the empty cLuc vector and the NPR3-nLuc or the empty cLuc vector and NPR4-nLuc construct. Two days after inoculation, $1 \mathrm{mM}$ luciferin was sprayed onto the inoculated leaves and chemiluminescence images were taken by a CCD camera 0 and $3 \mathrm{~h}$ after $1 \mathrm{mM}$ SA treatment. The fluorescence intensity was quantified using ImageJ.

Yeast two-hybrid. JAZs and JAZ1 derivatives were cloned into pGADT7. NPR3, NPR4 and NPR3 derivatives were cloned into pGBKT7 (ref. 50). These clones were transformed to the Saccharomyces cerevisiae strains AH109 and Y187, respectively, according to the Clontech yeast transformation protocol. After mating, diploid cells were selected on SD-Leu-Trp (SD-LW) plates. Single-positive colonies from each of the SD-LW plates were grown in the SD-LW liquid media for 1 day and then used for testing the interaction on SD-Leu-Trp-His-Ade (SD-LWHA) plates and SD-LWHA $+100 \mu \mathrm{M}$ SA plates.

Co-immunoprecipitation and pull down assays. For co-IP in N. benthamiana, NPR3 and NPR4 were cloned into the pK7FWG2 vector to create a C-terminal GFP fusion and JAZ1 was cloned into pEG203 to create an $\mathrm{N}$ terminal Myc fusion ${ }^{51,52}$ The NPR3-GFP, NPR4-GFP or GFP were co-expressed with Myc-JAZ1 in leaves of $N$. benthamiana for 2 days. Total proteins were extracted using the extraction buffer consisting $50 \mathrm{mM}$ Tris (pH 7.5), $150 \mathrm{mM} \mathrm{NaCl}, 0.1 \%$ Triton X-100, 0.2\% NP-40, $40 \mu \mathrm{M}$ MG115, and the protease inhibitor cocktail. $100 \mu \mathrm{M}$ SA was added to the protein samples and then incubated with GFP-Trap_A beads (Cat No. gta-10, ChromoTek) for $2 \mathrm{~h}$ at $4{ }^{\circ} \mathrm{C}$. For co-IP in Arabidopsis, 3-week-old plants of 35S:HA-JAZ1/35S:GFP, 35S:HA-JAZ1/35S:NPR3-GFP, 35S:HA-JAZ1/35S: NPR4-GFP were infiltrated with Psm ES4326/avrRpt2 at $\mathrm{OD}_{600 \mathrm{~nm}}=0.2$ together with $40 \mu \mathrm{M} \mathrm{MG115}$, and samples were collected 4 h.p.i. Then total proteins were extracted using the extraction buffer and incubated with GFP-Trap_A beads for $2 \mathrm{~h}$ at $4{ }^{\circ} \mathrm{C}$. After GFP-Trap_A beads were washed three times with the extraction buffer in the co-IPs performed in both N. benthamiana and Arabidopsis, proteins 
were eluted and denatured by heating in the protein loading buffer containing $100 \mathrm{mM}$ dithiothreitol at $95^{\circ} \mathrm{C}$ for $5 \mathrm{~min}$ before loading onto SDS-PAGE gels. The HA-JAZ1 or Myc-JAZ1 protein that co-precipitated with the GFP-tag proteins was detected by western blotting ${ }^{53}$ using the Myc (Cat No. sc-40, Santa Cruz Biotechnology; dilution, 1:1,000) or HA (Cat No. MMS-101P, Covance; dilution, 1:1,000) antibody. The HA-JAZ1, Myc-JAZ1 and GFP, NPR3-GFP or NPR4-GFP input levels were also measured by using HA, Myc or GFP (Cat No. JL-8, Living Colours; dilution, 1:2,000) antibody, respectively.

For the pull down assay, GST and GST-JAZ1 (ref. 54) were expressed in E. coli for $5 \mathrm{~h}$ at $25^{\circ} \mathrm{C}$ after IPTG induction. The NPR3-GFP and NPR4-GFP were transiently expressed in the NahG N. benthamiana transgenic line ${ }^{55}$ for 2 days, and treated with or without $1 \mathrm{mM} \mathrm{SA}$ or $100 \mu \mathrm{M} \mathrm{MeJA} 3 \mathrm{~h}$ before sample collection. After purification using Glutathione magnetic beads (Cat No. 88822, Thermo Scientific), GST and GST-JAZ1 were incubated with protein extract of NPR3-GFP or NPR4-GFP separately for $2 \mathrm{~h}$ at $4{ }^{\circ} \mathrm{C}$. Then beads were washed three times with the extraction buffer, and proteins were denatured by heating in the protein loading buffer containing $100 \mathrm{mM}$ dithiothreitol at $95^{\circ} \mathrm{C}$ for 5 min before loading onto SDS-PAGE gels. The NPR3-GFP and NPR4-GFP were detected by western blotting using the GFP antibody. The GST and GST-JAZ1 input levels were shown by Coomassie blue staining. Full versions of cropped blots are shown in Supplementary Fig. 19.

Hormone measurement. Phytohormones were extracted and quantified according to the method of Zeng et al. ${ }^{56}$ with some modifications. Four to six of the 3rd and 4th true leaves of 3-week-old plants ( $\sim 50 \mathrm{mg}$ ) were harvested before or after Psm ES4326/avrRpt2 infiltration at $\mathrm{OD}_{600 \mathrm{~nm}}=0.01$ at corresponding times. Samples were extracted at $4{ }^{\circ} \mathrm{C}$ overnight $(\sim 16 \mathrm{~h})$ using $0.45 \mathrm{ml}$ of ice cold methanol:water $(80: 20 \mathrm{v} / \mathrm{v})$ containing $0.1 \%$ formic acid, $0.1 \mathrm{gl}^{-1}$ butylated hydroxytoluene (BHT) spiked with abscisic acid $(\mathrm{ABA})-\mathrm{d}_{6}(100 \mathrm{nM})$ as an internal standard. Filtered plant extracts were injected onto a C18 column, and a gradient method starting with 9:1 (v/v) of solvent A ( $0.1 \%$ aqueous formic acid) and solvent B (100\% methanol) and increasing linearly to $100 \%$ solvent B was used for separation. For mass spectrometry, the capillary voltage, cone voltage and extractor voltage were set to $3.5 \mathrm{kV}, 25$ and $5 \mathrm{~V}$, respectively. Desolvation gas and cone gas were set to flow rates of 600 and $50 \mathrm{~h} \mathrm{~h}^{-1}$, respectively. Selected ion monitoring (SIM) was conducted in the negative electrospray channel for salicylic acid (SA; $m / z$ 137 $>93$ ), SA glucoside (SAG; $m / z 299.1>137$ ), abscisic acid (ABA; $m / z 263.1>153.1$ ), jasmonic acid (JA; $m / z 209.1>59$ ), JA-isoleucine (JA-Ile; $m / z 322.2>130.1$ ) and the internal ABA- $\mathrm{d}_{6}$ standard $(\mathrm{m} / z 269.1>159.1)$. Quan-Optimize software was used to determine the parent $>$ daughter SIM pairs used as well as the optimal source cone and collision energy voltages for each compound monitored. QuanLynx v4.1 software was used to determine analyte responses based on peak area integrations relative to the internal standard. Analytes were then quantified based on standard curves to determine the concentrations $(\mathrm{nM})$, which were converted to nanogram using the molecular weight of the compound and the extraction volume and normalized by sample fresh weight (FW) in gram. SAG was quantified based on the SA standard curve.

Pathogen infection. Psm ES4326/avrRpt2 was used to induce gene expression by infiltration into the 3 rd and 4 th true leaves of 3-week-old plants at $\mathrm{OD}_{600 \mathrm{~nm}}=0.2$ and samples were collected 4 h.p.i. The ion leakage assay was performed ${ }^{57}$ with six leaf discs suspended in $6 \mathrm{ml}$ of de-ionized water were used to measure the conductivity at $\mathrm{OD}_{600 \mathrm{~nm}}=0.01$. To check the effect of JA during ion leakage assays, $100 \mu \mathrm{M}$ MeJA or water was applied 3.5 h.p.i., and the leaf discs were collected 30 min later. To measure the bacterial growth, Psm ES4326/avrRpt2 was infiltrated into the 3rd and 4th true leaves of 3-week-old plants at indicated dosages, and in planta bacteria counts were monitored according to Cao et al. ${ }^{1}$.

Protein degradation. To analyse JAZ1 protein stability during ETI, $10 \mathrm{mM}$ $\mathrm{MgSO}_{4}$, Psm ES4326/avrRpt2 or Psm ES4326/avrRpt2 with $40 \mu \mathrm{M}$ MG115 was infiltrated into the $3 \mathrm{rd}$ and 4 th leaves of 3-week-old plants at $\mathrm{OD}_{600 \mathrm{~nm}}=0.2$. After $4 \mathrm{~h}, 0.2 \mathrm{~g}$ of the infiltrated leaves were collected for each sample. To measure the JAZ1 stability affected by SA in soil growing plants, $1 \mathrm{mM}$ SA were sprayed on 3-week-old plants for $4 \mathrm{~h}$. For the time course assay of JAZ1 stability, 2-week-old seedlings on MS plates were sprayed with $200 \mathrm{\mu g} \mathrm{ml}^{-1}$ CHX (Mock), $1 \mathrm{mM} \mathrm{SA}$ plus $200 \mu \mathrm{g} \mathrm{ml}^{-1}$ CHX (SA) or $100 \mu \mathrm{M}$ MeJA plus $200 \mu \mathrm{g} \mathrm{ml}^{-1}$ CHX (MeJA), and samples were collected at the corresponding times. Total protein was extracted using the extraction buffer mentioned above and denatured by heating at $95^{\circ} \mathrm{C}$ for $5 \mathrm{~min}$ after adding the protein loading buffer containing $100 \mathrm{mM}$ dithiothreitol. The HA-JAZ1 protein level was detected by western blotting using the HA antibody. $\beta$-Tubulin was also detected as an internal control using the $\beta$-Tubulin antibody (Cat No. sc-166729, Santa Cruz Biotechnology; dilution, 1:2,000) ${ }^{58}$. Full versions of cropped blots are shown in Supplementary Fig. 19.

Statistics. All data analyses were done using GraphPad Prism 6.00. Data are presented as mean \pm s.d. The Student's $t$-test was used to determine statistical significance for all pairwise comparisons and two-way analysis of variance (ANOVA) for data of the pathogen growth. The two-way ANOVA is a better statistical test for data with two independent variables. In our experiments, the ordinary two-way ANOVA was performed with no multiple comparisons and used colony forming units (the $\log _{10}$ c.f.u. in the $y$ axis) as dependent variables; days (treatments) after infiltration and genotypes as independent variables. Data distribution in this study was assumed to be normal, but not formally tested. The sample sizes were similar to those generally employed in the field.

Data availability. The authors declare that all data supporting the findings of this study are available within the article and its Supplementary Information files or are available on request from the corresponding authors.

\section{References}

1. Cao, H., Bowling, S. A., Gordon, A. S. \& Dong, X. Characterization of an arabidopsis mutant that is nonresponsive to inducers of systemic acquired resistance. Plant Cell 6, 1583-1592 (1994).

2. Wildermuth, M. C., Dewdney, J., Wu, G. \& Ausubel, F. M. Isochorismate synthase is required to synthesize salicylic acid for plant defence. Nature 414, 562-565 (2001).

3. Thomma, B. P. et al. Separate jasmonate-dependent and salicylate-dependent defense-response pathways in Arabidopsis are essential for resistance to distinct microbial pathogens. Proc. Natl Acad. Sci. USA 95, 15107-15111 (1998).

4. Trusov, Y. et al. Heterotrimeric G proteins facilitate Arabidopsis resistance to necrotrophic pathogens and are involved in jasmonate signaling. Plant Physiol. 140, 210-220 (2006).

5. van Wees, S. C., Chang, H. S., Zhu, T. \& Glazebrook, J. Characterization of the early response of Arabidopsis to Alternaria brassicicola infection using expression profiling. Plant Physiol. 132, 606-617 (2003).

6. Doares, S. H., Narvaez-Vasquez, J., Conconi, A. \& Ryan, C. A. Salicylic acid inhibits synthesis of proteinase inhibitors in tomato leaves induced by systemin and jasmonic acid. Plant Physiol. 108, 1741-1746 (1995).

7. Penacortes, H., Albrecht, T., Prat, S., Weiler, E. W. \& Willmitzer, L. Aspirin prevents wound-induced gene-expression in tomato leaves by blocking jasmonic acid biosynthesis. Planta 191, 123-128 (1993).

8. Van der Does, D. et al. Salicylic acid suppresses jasmonic acid signaling downstream of SCFCOI1-JAZ by targeting GCC promoter motifs via transcription factor ORA59. Plant Cell 25, 744-761 (2013).

9. Spoel, S. H. et al. NPR1 modulates cross-talk between salicylate- and jasmonate-dependent defense pathways through a novel function in the cytosol. Plant Cell 15, 760-770 (2003).

10. Spoel, S. H., Johnson, J. S. \& Dong, X. Regulation of tradeoffs between plant defenses against pathogens with different lifestyles. Proc. Natl Acad. Sci. USA 104, 18842-18847 (2007).

11. Thines, B. et al. JAZ repressor proteins are targets of the SCF(COI1) complex during jasmonate signalling. Nature 448, 661-665 (2007).

12. Kloek, A. P. et al. Resistance to Pseudomonas syringae conferred by an Arabidopsis thaliana coronatine-insensitive (coil) mutation occurs through two distinct mechanisms. Plant J. 26, 509-522 (2001).

13. Zander, M., La Camera, S., Lamotte, O., Metraux, J. P. \& Gatz, C. Arabidopsis thaliana class-II TGA transcription factors are essential activators of jasmonic acid/ethylene-induced defense responses. Plant J. 61, 200-210 (2010).

14. Li, J., Brader, G. \& Palva, E. T. The WRKY70 transcription factor: a node of convergence for jasmonate-mediated and salicylate-mediated signals in plant defense. Plant Cell 16, 319-331 (2004).

15. Mao, P., Duan, M., Wei, C. \& Li, Y. WRKY62 transcription factor acts downstream of cytosolic NPR1 and negatively regulates jasmonate-responsive gene expression. Plant Cell Physiol. 48, 833-842 (2007).

16. Zheng, X. Y. et al. Coronatine promotes Pseudomonas syringae virulence in plants by activating a signaling cascade that inhibits salicylic acid accumulation Cell Host Microbe 11, 587-596 (2012).

17. Yamada, S. et al. Involvement of OsJAZ8 in jasmonate-induced resistance to bacterial blight in rice. Plant Cell Physiol. 53, 2060-2072 (2012).

18. Tamaoki, D. et al. Jasmonic acid and salicylic acid activate a common defense system in rice. Plant Signal. Behav. 8, e24260 (2013).

19. Mur, L. A., Kenton, P., Atzorn, R., Miersch, O. \& Wasternack, C. The outcomes of concentration-specific interactions between salicylate and jasmonate signaling include synergy, antagonism, and oxidative stress leading to cell death. Plant Physiol. 140, 249-262 (2006).

20. Gassmann, W. \& Bhattacharjee, S. Effector-triggered immunity signaling: from gene-for-gene pathways to protein-protein interaction networks. Mol. Plant Microbe Interact. 25, 862-868 (2012).

21. Tsuda, K. \& Katagiri, F. Comparing signaling mechanisms engaged in patterntriggered and effector-triggered immunity. Curr. Opin. Plant Biol. 13, 459-465 (2010).

22. Kenton, P., J. M., L. A., Atzorn, R., Wasternack, C. \& Draper, J. (-)-Jasmonic acid accumulation in tobacco hypersensitive response lesions. Mol. Plant Microbe Interact. 12, 74-78 (1999).

23. Fu, Z. Q. et al. NPR3 and NPR4 are receptors for the immune signal salicylic acid in plants. Nature 486, 228-232 (2012) 
24. Rate, D. N. \& Greenberg, J. T. The Arabidopsis aberrant growth and death2 mutant shows resistance to Pseudomonas syringae and reveals a role for NPR1 in suppressing hypersensitive cell death. Plant J. 27, 203-211 (2001).

25. Sheard, L. B. et al. Jasmonate perception by inositol-phosphate-potentiated COI1-JAZ co-receptor. Nature 468, 400-405 (2010).

26. Fonseca, S. et al. (+)-7-iso-Jasmonoyl-L-isoleucine is the endogenous bioactive jasmonate. Nat. Chem. Biol. 5, 344-350 (2009).

27. Chini, A. et al. The JAZ family of repressors is the missing link in jasmonate signalling. Nature 448, 666-671 (2007).

28. Schweizer, F. et al. Arabidopsis basic helix-loop-helix transcription factors MYC2, MYC3, and MYC4 regulate glucosinolate biosynthesis, insect performance, and feeding behavior. Plant Cell 25, 3117-3132 (2013).

29. Song, S. et al. The Jasmonate-ZIM domain proteins interact with the R2R3-MYB transcription factors MYB21 and MYB24 to affect Jasmonateregulated stamen development in Arabidopsis. Plant Cell 23, 1000-1013 (2011).

30. He, Y., Chung, E. H., Hubert, D. A., Tornero, P. \& Dangl, J. L. Specific missense alleles of the arabidopsis jasmonic acid co-receptor COI1 regulate innate immune receptor accumulation and function. PLoS Genet. 8, e1003018 (2012).

31. Chung, H. S. et al. Regulation and function of Arabidopsis JASMONATE ZIM-domain genes in response to wounding and herbivory. Plant Physiol. 146, 952-964 (2008)

32. Kunkel, B. N., Bent, A. F., Dahlbeck, D., Innes, R. W. \& Staskawicz, B. J. RPS2, an Arabidopsis disease resistance locus specifying recognition of Pseudomonas syringae strains expressing the avirulence gene avrRpt2. Plant Cell 5, 865-875 (1993).

33. McHale, L., Tan, X., Koehl, P. \& Michelmore, R. W. Plant NBS-LRR proteins: adaptable guards. Genome Biol. 7, 212 (2006)

34. Heidrich, K. et al. Arabidopsis EDS1 connects pathogen effector recognition to cell compartment-specific immune responses. Science 334, 1401-1404 (2011).

35. Laudert, D. \& Weiler, E. W. Allene oxide synthase: a major control point in Arabidopsis thaliana octadecanoid signalling. Plant J. 15, 675-684 (1998).

36. Wasternack, C. \& Hause, B. Jasmonates: biosynthesis, perception, signal transduction and action in plant stress response, growth and development. An update to the 2007 review in Annals of Botany. Ann. Bot. 111, 1021-1058 (2013).

37. Wu, Y. et al. The Arabidopsis NPR1 protein is a receptor for the plant defense hormone salicylic acid. Cell Rep. 1, 639-647 (2012).

38. Manohar, M. et al. Identification of multiple salicylic acid-binding proteins using two high throughput screens. Front. Plant Sci. 5, 777 (2014).

39. Melotto, M. et al. A critical role of two positively charged amino acids in the Jas motif of Arabidopsis JAZ proteins in mediating coronatine- and jasmonoyl isoleucine-dependent interactions with the COI1 F-box protein. Plant J. 55, 979-988 (2008)

40. Santner, A. \& Estelle, M. The JAZ proteins link jasmonate perception with transcriptional changes. Plant Cell 19, 3839-3842 (2007).

41. Wager, A. \& Browse, J. Social network: JAZ protein interactions expand our knowledge of jasmonate signaling. Front. Plant Sci. 3, 41 (2012).

42. Shah, J. The salicylic acid loop in plant defense. Curr. Opin. Plant Biol. 6, 365-371 (2003).

43. Shim, J. S. et al. AtMYB44 regulates WRKY70 expression and modulates antagonistic interaction between salicylic acid and jasmonic acid signaling. Plant J. 73, 483-495 (2013).

44. Tsuda, K., Sato, M., Stoddard, T., Glazebrook, J. \& Katagiri, F. Network properties of robust immunity in plants. PLoS Genet. 5, e1000772 (2009).

45. Withers, J. et al. Transcription factor-dependent nuclear localization of a transcriptional repressor in jasmonate hormone signaling. Proc. Natl Acad. Sci. USA 109, 20148-20153 (2012).

46. Caldelari, D., Wang, G., Farmer, E. E. \& Dong, X. Arabidopsis lox3 lox4 double mutants are male sterile and defective in global proliferative arrest. Plant Mol. Biol. 75, 25-33 (2011).

47. Bates, D., Mächler, M., Bolker, B. \& Walker, S. Fitting linear mixed-effects models using lme4. J. Stat. Softw. 67, 1-48 (2015).

48. Chen, H. et al. Firefly luciferase complementation imaging assay for protein-protein interactions in plants. Plant Physiol. 146, 368-376 (2008).

49. Liu, L. et al. An efficient system to detect protein ubiquitination by agroinfiltration in Nicotiana benthamiana. Plant J. 61, 893-903 (2010).
50. Chien, C. T., Bartel, P. L., Sternglanz, R. \& Fields, S. The two-hybrid system: a method to identify and clone genes for proteins that interact with a protein of interest. Proc. Natl Acad. Sci. USA 88, 9578-9582 (1991).

51. Karimi, M., Inze, D. \& Depicker, A. GATEWAY vectors for Agrobacteriummediated plant transformation. Trends Plant Sci. 7, 193-195 (2002).

52. Earley, K. W. et al. Gateway-compatible vectors for plant functional genomics and proteomics. Plant J. 45, 616-629 (2006).

53. Burnette, W. N. 'Western blotting': electrophoretic transfer of proteins from sodium dodecyl sulfate--polyacrylamide gels to unmodified nitrocellulose and radiographic detection with antibody and radioiodinated protein A. Anal. Biochem. 112, 195-203 (1981).

54. Zentella, R. et al. O-GlcNAcylation of master growth repressor DELLA by SECRET AGENT modulates multiple signaling pathways in Arabidopsis. Genes Dev. 30, 164-176 (2016).

55. Ying, X. B. et al. RNA-dependent RNA polymerase 1 from Nicotiana tabacum suppresses RNA silencing and enhances viral infection in Nicotiana benthamiana. Plant Cell 22, 1358-1372 (2010).

56. Zeng, W. et al. A genetic screen reveals Arabidopsis stomatal and/or apoplastic defenses against Pseudomonas syringae pv. tomato DC3000. PLoS Pathog. 7, e1002291 (2011).

57. Mackey, D., Holt, 3rd B. F., Wiig, A. \& Dangl, J. L. RIN4 interacts with Pseudomonas syringae type III effector molecules and is required for RPM1-mediated resistance in Arabidopsis. Cell 108, 743-754 (2002).

58. Liu, N. K. \& Xu, X. M. beta-tubulin is a more suitable internal control than beta-actin in western blot analysis of spinal cord tissues after traumatic injury. J. Neurotrauma 23, 1794-1801 (2006).

\section{Acknowledgements}

We thank Rodoflo Zentella from Taiping Sun's lab at Duke University for the E. coli strain containing GST-JAZ1, and Huishan Guo from Institute of Microbiology, CAS for the NahG N. benthamiana transgenic line. This study was supported by grants from NIH 5R01 GM069594-11 the HHMI-GBMF (through Grant GBMF3032) to X. Dong, the Hargitt Fellowship to J. Withers, U.S.-Egypt Joint Board on Scientific and Technological Cooperation-STDF JUNIOR SCIENTIST DEVELOPMENT VISIT GRANT(ID: 3765) to F.-M. Sonbol, and NIH R01AI068718 and GBMF (GBMF3037) to S.Y. He.

\section{Author contributions}

L.L., J.W. and X.D. designed the research. F.-M.S. initiated the Y2H. B.H. performed the hormone measurements. Y.G. analysed the microarray data and M.M. analysed all the qRT-PCR data. J.Y. and S.Y.H. made all the JAZs constructs in the Gateway Donor vectors, and provided the coil-30 mutant, JAZ1 OE and jaz1 $\triangle j a s$ lines. L.L. performed the rest of the experiments. L.L. Y.G., J.W. and X.D. wrote the manuscript.

\section{Additional information}

Supplementary Information accompanies this paper at http://www.nature.com/ naturecommunications

Competing financial interests: The authors declare no competing financial interests.

Reprints and permission information is available online at http://npg.nature.com/ reprintsandpermissions/

How to cite this article: Liu, L. et al. Salicylic acid receptors activate jasmonic acid signalling through a non-canonical pathway to promote effector-triggered immunity. Nat. Commun. 7, 13099 doi: 10.1038/ncomms13099 (2016).

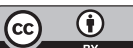

This work is licensed under a Creative Commons Attribution 4.0 International License. The images or other third party material in this article are included in the article's Creative Commons license, unless indicated otherwise in the credit line; if the material is not included under the Creative Commons license, users will need to obtain permission from the license holder to reproduce the material. To view a copy of this license, visit http://creativecommons.org/licenses/by/4.0/

(C) The Author(s) 2016 